\title{
ADVANTAGES OF A MODULAR DESIGN APPROACH FOR RADIOLOGICAL INSTRUMENTATION*
}

\author{
W. L. Bryan, S. R. Maddox, M. N. Ericson, C. L. Britton, M. S. Emery, and G. T. Alley \\ Oak Ridge National Laboratory \\ Instrumentation and Controls Division \\ P. O. Box 2008, Oak Ridge, TN 37831-6006
}

\begin{abstract}
The application of a modular design approach to the development and implementation of radiological instrumentation provides significant improvements over conventional instrument design in terms of capability, maintainability, and overall system cost. Two implementations utilizing this approach are discussed: a multifunction survey meter system developed for the U.S. Navy RADIAC program, and a stationary workplace monitoring system under development for use at Oak Ridge National Laboratory (ORNL). By designing modular, functionally partitioned hardware and software subsystems incorporating standard interfaces, it is possible to generate whole families of instrumentation systems in a building block fashion.
\end{abstract}

\section{INTRODUCTION}

Traditionally, radiological instrumentation development has been centered around the detector employed to perform the measurement of a specific radiation type. Desirable features, such as settable alarm points, scaler capability, background sampling and subtraction, and data logging have usually been added on. Unfortunately, manufacturability at minimum costs has generally been emphasized over maintainability, resulting in large support costs or limited service life. This developmental process has led to generations of task-specific instruments, primarily designed to measure a single form of radiation, incorporating mostly analog technology, and requiring a massive amount of maintenance and calibration effort in a large operation such as a national laboratory or a Navy fleet.

Today, with the ready availability of advanced detector technologies, low-cost microcontrollers, and computer-aideddesign tools, it is possible to take a system approach to the design of radiological instrumentation. The resulting design accommodates the necessary measurement functions for many forms of radiation, using a minimum amount of equipment and adding many extended capabilities previously not possible.

* Research sponsored in part by the U.S. Navy RADIAC Development Program under DOE Interagency Agreement No. 0531. 0531-A1 and in part by the Defense Advanced Research Projects Agency under DOE Interagency Agreement No. 1868-B135-A1. The Oak Ridge National Laboratory is operated by Martin Marietta Energy Systems, Inc. for the U. S. Department of Energy under Contract No. DE-AC05-84OR21400.

\section{A SURVEY METER SYSTEM}

The U.S. Navy is a major user of hand-held radiological survey instrumentation, in support of their nuclear propulsion, ordinance disposal, and contamination control missions. Their inventory consists of over 30 varieties of mostly analog technology, task-specific instruments, included under the general heading of "RADIAC" (radiation detection, indication and computation).

The staff of the ORNL Instrumentation and Controls division has worked with the Navy RADIAC program office for many years in the development of new detector technologies, instrument designs, and calibration and maintenance techniques. The goal of these efforts has been to improve measurement capabilities and to lower the overall costs of survey instrumentation support. One project that has recently moved from the lab to production development is the "Multi-Function RADIAC" (MFR), a new generation microcontroller based survey meter system designed to replace many types of existing single-function instruments by consolidating their functions into an integrated instrument and support system. The Navy will benefit from the life-cycle cost savings which will be made possible by this new system of instruments and support equipment.

The MFR is composed of a general-purpose handheld display unit with a built-in wide range gamma detector, plus a family of intelligent, attachable, radiation-specific probe units. The units communicate with each other via a serial data link using a standard message protocol. All system units contain a microcontroller subsystem (based on the Intel 8051 family), with non-volatile parameter storage (EEPROM) and serial I/O capabilities; they share many of the same software modules due to their common architecture (see Fig. 1, "MFR Architecture").

\section{A. Probe Units}

Each probe unit is responsible for control of its detector, generating necessary voltages from the standard battery power supplied by the display unit, and providing the required detector signal conditioning. It also applies stored calibration factors for unit conversion, performs value averaging, compares against stored alarm set points, checks its operating status, and reports to the display unit when polled. By using the internal processing and communication capability, probes 
can be individually calibrated following the procedure outlined in the Calibration and Maintenance Advantages section below.

A variety of MFR compatible prototype probe units have been developed or adapted to demonstrate the advantages of a modular design approach. These include an energy compensated, six decade beta/gamma end-window Geiger Muller tube probe, a $100 \mathrm{~cm}^{2}$ alpha scintillation probe, a light weight, mixed energy neutron indicating probe and a REM responding neutron "snoopy" probe.

The development of the initial MFR beta/gamma probe was the most time consuming; however, its development became the basis for modules which were utilized in the design of future probe units. The basic microcontroller and high voltage converter modules are identical from one MFR probe to another. Only the circuits for different detector signal interfaces are probe specific. Software development for the initial probe unit was likewise time consuming, but all the software modules for the basic time management kernal, communications protocol handling and data averaging were reused in other probe units as well as the display unit. Only. the data conversion software associated with different detector types is probe specific. After the initial probe unit was completed, subsequent probe development required less than one tenth the effort. Software development required to support a new probe unit type now generally takes days, versus months for the initial module development.

\section{B. Display Unit}

The display unit contains a software programmable dotmatrix LCD panel, a programmable tone generator ASIC developed at ORNL [1], and a battery power supply. The display unit acts as the MFR bus master and is responsible for polling the attached probe unit(s) to determine the probe type, status, and units of display at power up. It then begins continuous polling of the probe(s) for the current measurement, alarm and status information, and updates the display. The display provides indication of: measurement value (in large block digits), units of measurement (e.g., $\mathrm{Gy} / \mathrm{hr}$ ), decade and percent of decade (via a multi-segment bar graph), type of radiation (e.g., GAMMA), alarm and status condition (WARNING, ALARM, OVER, INOP), and battery level (percent remaining).

Since the display unit obtains all of the probe specific information from the probe unit itself, it remains generic and automatically configures the display to suit the measurement task addressed by a particular probe. Since each probe unit retains all information regarding units of measurement, calibration factors, and alarm set points, any probe unit can be attached to any display unit and operate without requiring probe specific calibration or display unit adjustment.

\section{Equipment Consolidation}

An entire survey "kit" can be created with one display unit and a set of appropriate radiation probe units which accomplish most if not all types of radiation measurements. By using a common display unit and a limited number of independently calibrated probes for multiple radiation type survey tasks, operational procedure generation and operator training costs can be considerably reduced. This also reduces the costs of inventory, maintenance, shipping, and storage compared to the support of the large number of currently required task-specific instruments with dedicated displays.

\section{A WORKPLACE MONITORING SYSTEM}

The Oak Ridge National Laboratory (ORNL) is a major research center for nuclear reactor design, materials research

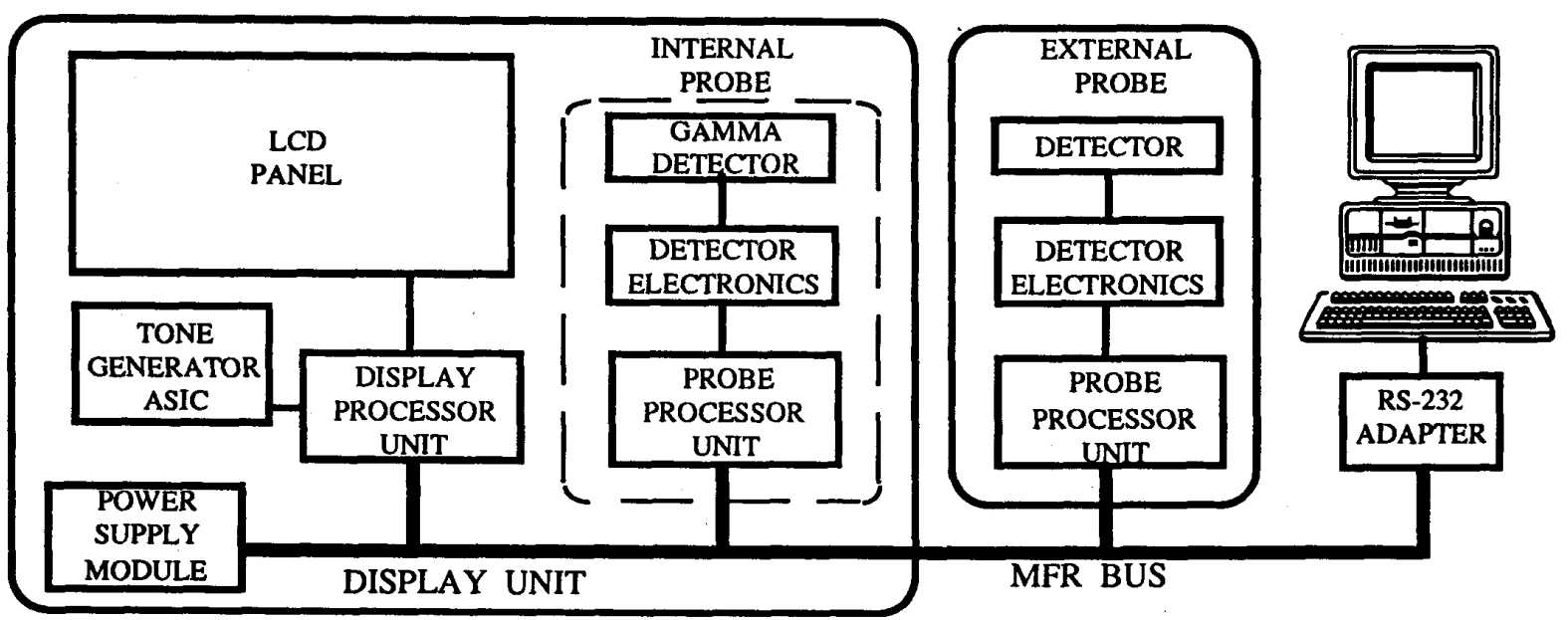

Fig. 1. MFR Architecture 
and isotope production. ORNL utilizes a large population of stationary, workplace monitoring instruments due to the presence of many potential sources of radiation in these work areas. The inventory includes constant air monitor systems (alpha and beta/gamma), gamma area monitors, hand and foot monitors, contamination friskers (alpha and beta/gamma), neutron criticality monitors, and others. Due to the age of the plant, some of the instruments still in service utilize 1960s era technology, most record keeping is done with paper strip chart recorders, instrument calibration is still a manual, laborintensive job, and the current facility protection and alarm systems employ aging relay logic.

A new system for centralized monitoring, alarming, and record keeping is currently being developed by applying the same modular design concepts, and in some cases the same reusable subsystems, generated during the evolution of the MFR handheld survey meter system described previously.

New detector systems and instruments incorporating modular design features are being designed and procured. These include state-of-the-art alpha air monitoring subsystems, friskers, portal monitors, and others. They are intended to satisfy regulatory requirements, and to provide the types of life-cycle cost benefits associated with streamlincd maintenance and calibration activities.

Each instrument, regardless of type, is to be equipped with a standard "instrument interface" unit, which will provide the functions of signal conditioning, unit conversion, averaging, measurement display, local alarm indication and data logging, automated check source activation, operational testing and high-speed network communication using a standard protocol and command set. The interface unit is based on an Intel 8044 microcontroller which supports the "BITBUS" communication protocol plus a large non-volatile EEPROM memory for local record storage, a battery backed-up real time clock, an LCD panel for measurement and status display, click and alarm tone generation, and fiber optic transceivers for network connection (see Fig. 2a \& 2b, "Instrument Interface Unit - Facility Instrument Network Architecture").

All instruments in a given process area (or facility), which may span several nearby buildings, are to be interconnected by means of a multi-drop network to a "facility computer" located in the facility Health Physics office. From this central station, the facility Health Physicist can view the current alarm status and measurement data from all the instrument locations in the facility via a graphical display, which shows the instruments relative to a floor plan of the facility and uses color to indicate status (green, yellow, magenta, red, white).

Every instrument on the network is polled at frequent intervals, and in the event of a serious condition (multiple instrument alarms, or a network failure), a central facility alarm is triggered and the plant emergency response center is notified. Radiation measurement data from every instrument is continuously obtained and recorded at the facility computer as required to meet record keeping requirements.

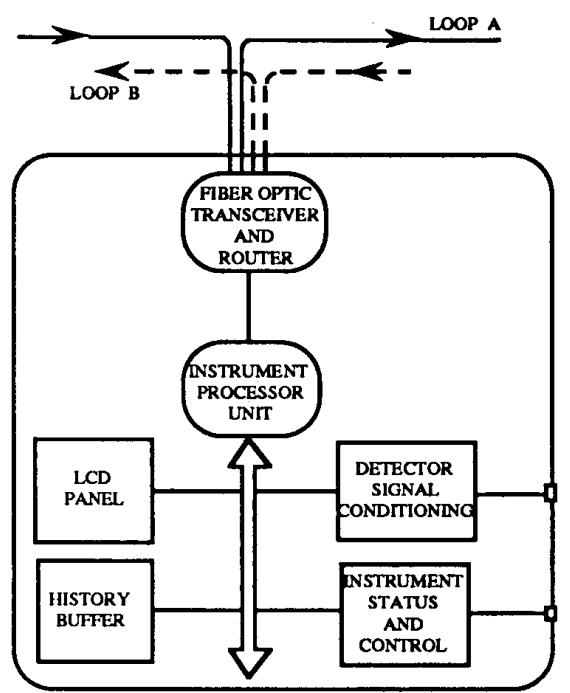

Fig. 2a. Instrument Interface Unit

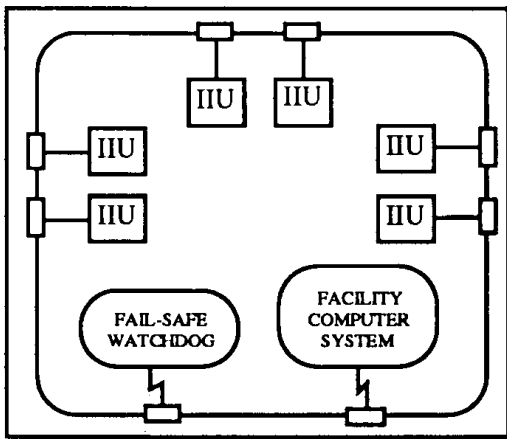

Fig. 2b. Facility Instrument

Network Architecture

Calibration history information, alarm set points and other stored parameters may be remotely retrieved from (or downloaded to) any instrument by way of its network connection. The calibration status of all active instruments is periodically checked by the facility computer, and the facility Health Physicist is automatically notified of upcoming calibration due dates.

The multi-drop facility instrument network is implemented using a dual, counter-directional fiber optic loop, with one loop being the primary or active loop and the other loop normally in a standby mode. In the event of a failure on the primary loop, the standby loop is activated. If a double failure occurs (both loops broken), then individual nodes on both sides of the failure "loopback" using both loops, thus preserving a continuous path (see Fig. 3 "Fault Tolerant Facility Network). A network watchdog node is also incorporated which listens for continuous network traffic. If 
the normal polling traffic stops, it triggers the facility alarms, acting as a fail-safe device for the facility computer and the instrument network.
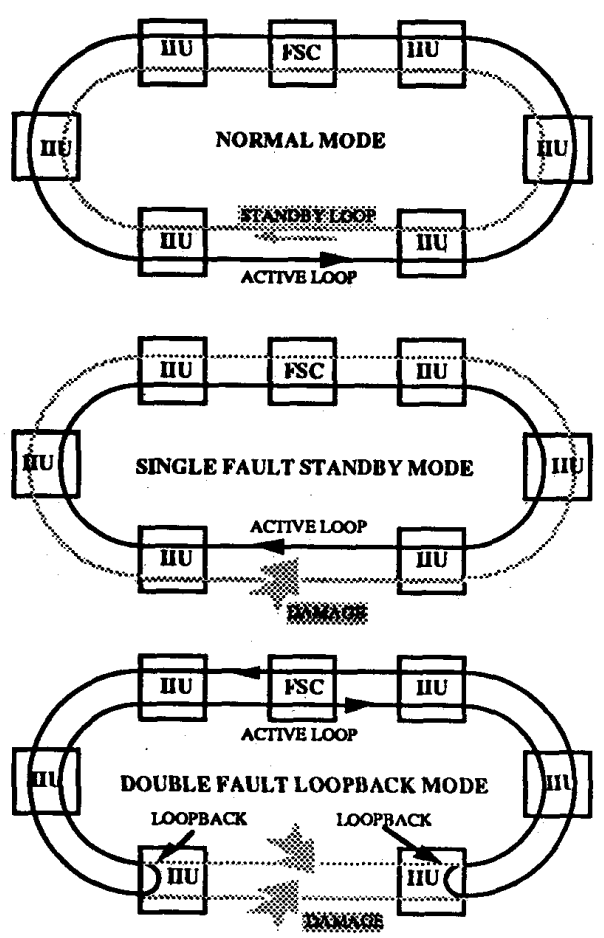

Fig. 3. Fault Tolerant Facility Network

The system as a whole is designed such that facility operational safety will not be compromised, new instruments can easily be added to the inventory without requiring system redesign or modification of the standard interface, maintenance and calibration costs will be kept to a minimum, and greatly increased record keeping and reporting capabilities will be added. A benchtop proof-of-concept model of this system, utilizing existing ORNL stationary instruments, has been developed and demonstrated. Instruments which were interfaced to the demonstration facility network included alpha and beta/gamma air monitors, gamma area monitors, alpha friskers and beta friskers. The single and dual fault tolerance capabilities of the fiber-optic facility network was verified.

\section{DEVELOPMENT ADVANTAGES}

For the instrument developer, the modular design approach offers many benefits in terms of devclopment cost, system testability, and time-to-market. By functionally partitioning the various instrument system functions, and developing an inventory of reusable hardware and software subsystems to support those functions, new instrument systems can be readily generated which incorporate the desired features.
A typical instrument design may incorporate some or all of the following functional subsystems: a radiation specific detector assembly, battery power supply, high-voltage power supply, signal conditioning circuitry, microcontroller unit, non-volatile parameter memory, data logging memory, liquid crystal display (LCD) panel, tone generating circuit, self-test routines, check source control, display generation routines, unit conversion and averaging algorithms, communication routines, etc.

Using this building block approach leads to more reliable designs which cost less to verify, since new designs are based upon pre-tested subsystems which can be reused with high confidence that they will perform as required in the overall system. In addition, the subsystem inventory can be continuously improved by incorporating new detector technologies, electronic technologies, display technologies, software algorithms, and so on without having to sacrifice the investment made in other already developed subsystems.

In addition, the use of a smaller number of more capable, solid-state components, including microcontrollers, can greatly reduce the amount of design effort required to incorporate new instrument features. Many of these functions may be implemented in software (averaging algorithms, customized displays, selectable operating modes, etc.). High-level tools, including PC-based cross compilers, in-circuit emulators, program librarians, and other common software development facilities, are readily available. The embedded processor approach allows for rapid prototyping and incorporation of new features without a major redesign effort.

\section{CALIBRATION AND MAINTENANCE ADVANTAGES}

Obviously, for a large operation such as a national laboratory, which has literally thousands of portable and stationary radiation monitoring instruments, the calibration and maintenance functions required becomes an enormous cost relative to the initial procurement cost. These costs are magnified by the number of different task-specific instruments of varying technology that have been introduced into the inventory over the past 40 years, each requiring specific calibration and maintenance procedures with associated training and support costs.

\section{A. Instrument Maintenance}

Just as in the early days of consumer electronics, before the advent of inexpensive board-level replacement, these older generation instruments require component level troubleshooting and repair. As time goes by, the components become harder to obtain, labor costs (including training) increase, and a backlog of instruments requiring service grows. 
By contrast, modular designs lend themselves to the practice of subsystem replacement and rapid return to service. Parts inventory costs can be reduced because of common subsystems used by various instruments. Labor costs can be reduced, with less dependence on highly skilled troubleshooting. A requirement for modular design of newly acquired instruments should have a significant positive effect on the maintenance problem as the older instruments are gradually retired from service.

In addition, instruments equipped with microcontrollers can be programmed to perform self test routines to assist in the location of faulty modules and subsystems. They can also be made to operate in a diagnostic mode as nceded to check detector performance, can communicate with automated test equipment, and so on.

\section{B. Instrument Calibration}

Calibration of older generation instruments is a tedious and sometimes hazardous matter of exposing the instrument to a radiation source, tweaking analog adjustments on the instrument, and iterating until an acceptable reading is obtained. As much as two hours or more can be spent adjusting a single instrument on an open source range.

With the advent of microcontroller based instrument designs, however, this procedure has been greatly simplified. Instead of requiring a tedious manual procedure which may expose a technician to an undesirable dose of radiation, calibration may now be performed remotely by means of a communication link from the instrument to a computer system. Once the instrument is positioned in the calibration range (or calibrator enclosure), it is exposed to a ficld of the desired strength, a measurement is obtained by polling the instrument, and a conversion factor is calculated and downloaded to the instrument where it is stored in a non-volatile memory for subsequent use. An automatic log of the instrument serial number, calibration date and time, and technician ID is maintained in the calibration computer system with records generated as required. In this fashion, the entire calibration process is reduced from hours to minutes.

\section{PERFORMANCE ADVANTAGES}

Some of the performance advantages provided by modular instrument design have already been suggested. In particular, the incorporation of microcontroller technology has a major impact on the level of instrument performance and capability.

Older generation instruments provide only a limited set of the desirable features (averaging constants, alarm set points, display ranges, etc.) and are not generally expandable to include others. They tend to be task-specific and operate in a stand-alone mode, with minimal remote reporting capability, if any.

Microcontroller based instruments, however, have the ability to employ versatile, readily modifiable software algorithms for instrument control, unit conversion, graphical display, parameter storage, and long-distance communication. Thus, a whole new class of instrument capabilities is made available.

A reusable microcontroller subsystem can be developed which provides a flexible, configurable platform to support various system functions and features with minimal changes (if any) required in the hardware. Such a subsystem would include the desired processor, scratch pad memory, program storage, parameter storage, serial $\mathrm{I} / \mathrm{O}$, counter/timers, etc.

\section{CONCLUSION}

A modular approach to the design of new-generation radiological instrument systems, with emphasis on testable, reusable, upgradeable hardware and software subsystems together with solid-state and microcontroller technology, has been successfully employed in several recent applications. This approach produces real benefits in terms of instrument system development time, functional versatility, operational reliability, maintainability and overall life-cycle cost.

\section{REFERENCES}

[1] C. L. Britton, Jr., G. T. Alley, W. L. Bryan, M. S. Emery, M. N. Ericson, D. W. Bouldin, D. F. Newport, "Tool Integration and Enhancement for Standard Cell Designs," Proceedings, 1989 VLSI Education Conference and Exposition, pp. 97-104, July 1989. 